\title{
Angioimmunoblastic T-cell lymphoma combined with Hodgkin's lymphoma:a case report
}

Xiaohan $\mathrm{Gao}^{1}$, Jing $\mathrm{Li}^{2}$, Lin Kang ${ }^{1}$, Qiang $\mathrm{Wei}^{1}$, Jie $\mathrm{Li}^{1}$, Jie Yang ${ }^{1}$, Hongling $\mathrm{Hao}^{1}$, and Yan $\mathrm{Li}^{1}$

${ }^{1}$ Hebei General Hospital

${ }^{2}$ Hebei Province Chinese Medicine Hospital

August 21, 2021

\begin{abstract}
Angioimmunoblastic T-cell lymphoma (AITL) is a special subtype of peripheral T-cell lymphoma, Histopathological examination is the main basis for the diagnosis of the disease, but the diagnosis is challenging. The study has shown that combining immunohistochemistry and gene rearrangement can increase the diagnostic accuracy
\end{abstract}

Angioimmunoblastic T-cell lymphoma combined with Hodgkin's lymphoma:a case report

Xiao-han Gao ${ }^{\mathrm{a}}$, Jing $\mathrm{Li}^{\mathrm{b}}$, Lin Kang ${ }^{\mathrm{c}}$, Qiang Wei ${ }^{\mathrm{d}}$, Jie $\mathrm{Li}^{\mathrm{a}}$, Jie Yang ${ }^{\mathrm{a}}$, Hong-ling Hao ${ }^{\mathrm{a}}$ and Yan $\mathbf{L i}^{\mathrm{a}, *}$

*Correspondence to

Yan Li, Ph.D.

Department of Hematology,

Hebei General Hospital,

No. 348, HepingWest Road,

Shijiazhuang 050051, Hebei, China

Tel: $(+86) 18931866300$;

Email: 18931866300@163.com

\begin{abstract}
Angioimmunoblastic T-cell lymphoma (AITL) is a special subtype of peripheral T-cell lymphoma with a poor prognosis, clinical manifestations combinationed with immunohistochemistry, gene rearrangement and so on can increase the diagnostic accuracy.

Introduction: Angioimmunoblastic T-cell lymphoma (AITL) is a special subtype of peripheral T-cell lymphoma, Histopathological examination is the main basis for the diagnosis of the disease, but due to lack of specific pathological characteristics, the diagnosis is challenging. The study has shown that combining immunohistochemistry and gene rearrangement can increase the diagnostic accuracy.
\end{abstract}

Patient Concerns: A 56-year-old male patient was admitted to the hospital due to skin redness and itching for 4 months. 
Diagnoses: The patient was diagnosed with AITL with Hodgkin's lymphoma based on lymph node biopsy, immunohistochemistry, gene rearrangement and positron emission tomography computer tomography

Keywords: follicular lymphoma, myelofibrosis, immunohistochemistry, cytokines

\section{Introduction}

Angioimmunoblastic T-cell lymphoma (AITL) is a subtype of peripheral T-cell lymphoma with poor prognosis ${ }^{1}$ accounting for 1-2\% of non-Hodgkin's lymphoma and 15-20\% of peripheral T-cell lymphoma. The median age is approximately 65 years. The clinical manifestations mostly include fever, weight loss, urticaria, papules, red nodules, and skin lesions ${ }^{2}$ Due to the abnormal proliferation activity of B cells, AITL is often accompanied by autoimmune disorders, such as hemolytic anemia and hypergammaglobulinemia. Studies have shown the Epstein-Barr virus (EBV) to play an important role in the pathogenesis of AITL; EBV can stimulate the activation of helper $\mathrm{T}$ cells, thereby leading to the occurrence of tumors. While histopathological examination forms the basis for diagnosis of the disease, the latter still remains challenging due to the lack of specific pathological characteristics. The current study suggested that combination of immunohistochemistry and gene rearrangement can increase diagnostic accuracy. CD10 and CXCL13 are specifically expressed in AITL and can be used as characteristic markers for the diagnosis of AITL. The clonal rearrangement of IgH gene and TCR $\curlyvee$ would also be of great significance in the diagnosis.

\section{Case Report}

A 56-year-old man was admitted to the hospital with Hodgkin's lymphoma. The patient received ABVD and AVD chemotherapy, and all details of unsatisfactory treatment effect were collected, wherefrom angioimmunoblastic T-cell lymphoma and focal classical Hodgkin's lymphoma (compound lymphoma) were revealed. CD21 was found to shrink and destroy the FDC network, CD20+; PAX-5+; CD3+; Ki-67+: 20\%-30\%; CD10-; BCL-6+; MUM-1+; PD-1+; CXCL-13-; CD30+; CD15-. Molecular detection: TCR $\beta D B+J \beta 1 / 2$ showed monoclonal rearrangement, and $\mathrm{V} \beta+\mathrm{J} \beta 2$ showed oligoclonal rearrangement.

The patient was admitted to the hospital owing to skin redness and itching for 4 months. Physical examination revealed extensive redness, swelling, and rough skin on the head, neck, and limbs. There were multiple enlarged lymph nodes in the right armpit and bilateral groin. The largest one was approximately $4 \mathrm{~cm}$ in diameter, with a smooth surface, no tenderness, and good mobility. The laboratory examination results are presented in Table 1. At first, a proliferative disease of the lymphatic system was suspected; a punch biopsy was performed, whose histological diagnosis documented classic Hodgkin's lymphoma, lymphocyterich type, with molecular detection: EBER (+). In addition, CD3+ lymphocytes occupied $96.1 \%$, positively expressing CD3 and CD5, whereas some expressed TCRrd, and a small number expressed CD8, as per immunohistochemistry . Multiple organs with lymph node infiltration were detected using radiological imaging. Interestingly, the patient presented with skin redness. The patient completed the ABVD (doxorubicin hydrochloride liposome $40 \mathrm{mg}$ ivgtt $\mathrm{d} 1,15$; bleomycin $10 \mathrm{mg} / \mathrm{m} 2$ iv d1, 15; vindesine $4 \mathrm{mg}$ iv d1, 15; dacarbazine $375 \mathrm{mg} / \mathrm{m} 2$ iv d1,15) chemotherapy, followed by AVD in the second course. However, he tended to have a poor performance status, and skin pruritus symptoms were aggravated. Immunohistochemical examination of the skin revealed CD20 +, PAX-5 +, CD3 +, Ki-67 + (20-30\%), CD10-, BCL-6 +, MUM-1 +, PD-1 + , CXCL-13-, CD30 +, and CD15-(Fig. and 1,2). TCR $\beta D B+J \beta 1 / 2$ showed monoclonal rearrangement, and $\mathrm{V} \beta+\mathrm{J} \beta 2$ showed oligoclonal rearrangement upon molecular examination with PTPRD gene mutation. Pathological diagnosis was non-Hodgkin peripheral (mature) T-cell lymphoma, prone to angioimmunoblastic T-cell lymphoma. Due to the angioimmunoblastic T-cell lymphoma with Hodgkin's lymphoma, it was decided that the patient would be followed-up with EDOCH chemotherapy; however, unfortunately, he died of lung infection.

\section{Discussion}

AITL is a special subtype of peripheral T-cell lymphoma that originates from follicular helper T cells (TFH), often with fever, night sweats, weight loss, lymphadenopathy, skin rash, and other clinical manifestations ${ }^{3}$ 
Skin involvement is one of the most common extranodal manifestations of the disease ${ }^{4}$ Due to the heterogeneity of AITL, most cases do not get diagnosed until weeks or months after the onset of symptoms.

While the diagnosis of AITL relies on lymph node biopsy, some patients may be diagnosed after 2-3 lymph node biopsies. The pathological features include the destruction of lymph node structure, the tumor cells being mainly of medium size, the cytoplasm being lightly stained or transparent, the nucleus being generally round or oval, and the cell being atypical . In the background of inflammatory cells, such as eosinophils, lymphocytes, and plasma cells, large cells of varying numbers are scattered. The TFH phenotype is positive for CD3, CD4, and CD10 ${ }^{5}$ Most CD5 and CD7 expression are absent ${ }^{6}$ CD30 is found in $20 \%$ of patients ${ }^{7}$ Cytoplasmic CXCL13 is almost uniformly expressed, and is specific for AITL ${ }^{8}$ TFH expression of PD-1, ICOS, BCL-6, and CD200 can be distinguished from that in benign lymphoproliferative diseases and PTCL subtypes. Approximately $60 \%$ of patients have TCR gene rearrangements, as seen in TC $\beta$, TCD, and TCG rearrangements, whereas some patients have IgH gene rearrangements. In recent years, with advanced research on genomics, AITL has been found to have higher TET2, RHOA, IDH2, and DNMT3A mutations, especially TET2 mutations, which are related to poor prognosis ${ }^{9}$ However, AITL currently lacks a systematic method of identification, and hence its diagnosis is still challenging.

The occurrence of AITL is highly correlated with EBV infection. EBV-infected B cells can transmit EBV protein signals on their surface to $\mathrm{T}$ cells through major histocompatibility complex II (MHCII) molecules when TFH cells interact with B cells. Cells up-regulate the expression of CD ligands, provide antigen and costimulatory signals for $\mathrm{T}$ cell activation, and promote the secretion of chemokine CXCL13 ${ }^{10}$ which subsequently leads to B cell activation. Laforga et $\mathrm{al}^{11}$ had proposed the hypothesis that EBV promotes the proliferation of B cells in AITL. They believed that due to the effect of EBV, CD8-positive T cells are immunosuppressed, leading to oversight of EBV-positive B cells. EBV-infected B cells lose control and may be polyclonal, oligoclonal, or monoclonal. If the immunoglobulin structure is broken, B cells can have three outcomes: 1. proliferation resembling that of RS cells; 2. proliferation resembling that in CHL; or 3. it might develop into CHL.

In the 2008 edition of the 'WHO Classification of Tumors of Hematopoietic and Lymphoid Tissues', RS-like cells were mentioned, for the first time, to be seen in early AITL ${ }^{12}$; AITL and CHL also have lymph node structure destruction, and plasma cell, tissue cell, and other inflammatory cell infiltration, in addition to a correlation with EBV infection ${ }^{13}$ thereby confirming the dependence of AITL and CHL on immunophenotypes only. The immunophenotype of CHL includes CD15+, CD30+, PAX-5 weakly positive, CD3-, CD20(mainly), CD45-, and CD79a-, which combined with RS cells, help in the diagnosis ${ }^{14,15}$ CHL and AITL are two types of lymphoma with extremely different prognoses. While CHL has good prognosis, with a 5-year overall survival (OS) rate $>80 \%^{16}$ AITL has poor prognosis. A retrospective analysis had found (after CHOP chemotherapy in elderly patients with AITL) the CR rate to be $25 \%$, and the median OS to be only 14.9 months. At present, ABVD is the first-line treatment for CHL, and most patients benefit from it. However, AITL progresses rapidly with high mortality rate. Therefore, patients with newly diagnosed lymphoma should be verified based on clinical phenotype, pathological morphology, and immunohistochemistry for better and successful.

\section{The Key Clinical Message}

Clinical manifestations combinationed with immunohistochemistry, gene rearrangement can increase the diagnostic accuracy of lymphoma.

\section{Acknowledgments}

We acknowledge the cooperation of the patient's relatives. Published with written consent of the patient's relatives.

\section{Conflict of Interest Statement}

The authors report no conflict of interest 


\section{Author Contributions}

Xiao-han Gao, Jing Li,Lin Kang, Qiang Wei, Jie Li,Jie Yang and Hongling Hao:

collected and recorded the clinical data.

Xiao-han Gao and Yan Li: analyzed the case, wrote the manuscript and performed the

critical revision.

\section{Ethics Statement}

Written consent was obtained from the patient's relatives ahead of submission.

\section{References}

1. Swerdlow, S.H., E. Campo, S. A. Pileri, et al. 2016. The 2016 revision of the World Health Organization classification of lymphoid neoplasms. Blood 127(20):2375-2390.

2. Lachenal, F., F. Berger, H. Ghesquières, et al. 2007. Angioimmunoblastic T-cell lymphoma: clinical and laboratory features at diagnosis in 77 patients. Medicine (Baltimore) 86(5):282-292.

3. Xie, C., X. Li, H. Zeng, and W. Qian. 2020. Molecular insights into pathogenesis and targeted therapy of peripheral T cell lymphoma. Exp Hematol Oncol 9(1):30.

4. Botros, N., L. Cerroni, A. Shawwa, et al. 2015. Cutaneous manifestations of angioimmunoblastic T-cell lymphoma: clinical and pathological characteristics. Am J Dermatopathol 37(4):274-283.

5. Lunning, M. A., and J. M. Vose. 2017. Angioimmunoblastic T-cell lymphoma: the many-faced lymphoma. Blood 129(9):1095-1102.

6. Rodríguez-Cortés, J., and T. Palomero. 2016. The curious origins of angioimmunoblastic T-cell lymphoma. Curr Opin Hematol 23(4):434-443.

7. de Leval, L., D. S. Rickman, C. Thielen, et al. 2007. The gene expression profile of nodal peripheral T-cell lymphoma demonstrates a molecular link between angioimmunoblastic T-cell lymphoma (AITL) and follicular helper T (TFH) cells. Blood 109(11):4952-4963.

8. Grogg, K. L., A. D. Attygale, W. R. Macon, E. D. Remstein, P. J. Kurtin, and A. Dogan. 2006. Expression of CXCL13, a chemokine highly upregulated in germinal center T-helper cells, distinguishes angioimmunoblastic T-cell lymphoma from peripheral T-cell lymphoma, unspecified. Mod Pathol 19(8):1101-1107.

9. Hopfinger, G., and P. Staber. 2019. Current standard in diagnostic and therapy of peripheral T-cell lymphoma. Dtsch Med Wochenschr 144(20):1400-1404.

10. Dunleavy, K., H. W. Wilson, and E. S. Jaffe. 2007. Angioimmunoblastic T cell lymphoma: pathobiological insights and clinical implications. Curr Opin Hematol 14(4):348-353.

11. Laforga, J. B., J. M. Gasent, and M. Vaquero. 2010. Potential misdiagnosis of angioimmunoblastic T-cell lymphoma with Hodgkin's lymphoma: a case report. Acta Cytol 54(5):840-844.

12. Gao, X., W. Huang, W. Li, J. Xie, Y. Zheng, and X. Zhou. 2015. Clinicopathologic analysis of angioimmunoblastic T-cell lymphoma with Hodgkin/Reed-Sternberg-like cells. Chin J Pathol 8(44):553-558.

13. Parekh, V., and D. Peker. 2015. EBV-related primary splenic lymphocyte-depleted classical Hodgkin lymphoma. J Clin Pathol 68(11):947-950.

14. Ferrarini, I., A. Rigo, C. Visco, M. Krampera, and F. Vinante. 2020. The evolving knowledge on $\mathrm{T}$ and NK cells in classic Hodgkin lymphoma: insights into novel subsets populating the immune microenvironment. Cancers (Basel) 12(12):3757.

15. Connors, J. M. 2020. Hodgkin lymphoma: outsmarting HRS cells. Blood 136(21):2362-2364.

16. Moccia, A. A., S. Aeppli, S. Güsewell, et al. 2021. Clinical characteristics and outcome of patients over 60 years with Hodgkin lymphoma treated in Switzerland. Hematol Oncol 39(2):196-204.

Table 1. The laboratory findings

\begin{tabular}{lll}
\hline CBC & WBC & $15.00 \times 10^{\wedge} 9 / \mathrm{L}$ \\
& LY & $5.12 \times 10^{\wedge} 9 / \mathrm{L}$
\end{tabular}




\begin{tabular}{lll} 
& HGB & $151 \mathrm{~g} / \mathrm{L}$ \\
32-Microglobulin & PLT & $292 \times 10^{\wedge} 9 / \mathrm{L}$ \\
Biochemistry & TP & $4.288 \mathrm{ug} / \mathrm{mL}$ \\
& GLO & $53.5 \mathrm{~g} / \mathrm{L}$ \\
& LDH & $22.3 \mathrm{~g} / \mathrm{L}$ \\
& HBDH & $323.9 \mathrm{U} / \mathrm{L}$ \\
& TG & $222.4 \mathrm{U} / \mathrm{L}$ \\
Coombs test & Direct Coombs test & $1.84 \mathrm{mmol} / \mathrm{L}$ \\
& Indirect Coombs test & negative \\
Tumor Marker & CA 125 & $125.100 \mathrm{U} / \mathrm{mL}$ \\
& VEGF & $178.4 \mathrm{pg} / \mathrm{mL}$ \\
\hline
\end{tabular}

CBC:complete blood count;WBC:white blood cell;LY:lymphocyte cell; HGB:hemoglobin; PLT:plate;TP:Total protein;GLO:Globulins;LDH:Lactate dehydrogenase;HBDH:GHB dehydrogenase TG:Triglyceride;VEGF:Vascular endothelial growth factor

\section{Hosted file}

Zhang-figures.pdf available at https://authorea.com/users/431237/articles/534744angioimmunoblastic-t-cell-lymphoma-combined-with-hodgkin-s-lymphoma-a-case-report 